\title{
A Chest Patch for Continuous Vital Sign Monitoring: Clinical Validation Study During Movement and Controlled Hypoxia
}

Carlos Morgado Areia ${ }^{1,2}$, MSc, PT; Mauro Santos ${ }^{2,3}$, DPhil; Sarah Vollam ${ }^{1,2}$, MSc, RN; Marco Pimentel ${ }^{2,3}$, DPhil; Louise Young ${ }^{1,2}, \mathrm{RN}$; Cristian Roman ${ }^{2,3}, \mathrm{PhD}$; Jody Ede ${ }^{1,2}$, MSc, RN; Philippa Piper ${ }^{1}$, BSc; Elizabeth King ${ }^{4}$, BSc; Owen Gustafson $^{4}$, MSc; Mirae Harford ${ }^{1,2}$, MD; Akshay Shah ${ }^{5}$, FRCA; Lionel Tarassenko ${ }^{2,3}$, MA, DPhil; Peter Watkinson ${ }^{1,2,6}, \mathrm{MD}$

\footnotetext{
${ }^{1}$ Critical Care Research Group, Nuffield Department of Clinical Neurosciences, University of Oxford, Oxford, United Kingdom

${ }^{2}$ National Institute for Health Research, Biomedical Research Centre, Oxford, United Kingdom

${ }^{3}$ Institute of Biomedical Engineering, Department of Engineering Science, University of Oxford, Oxford, United Kingdom

${ }^{4}$ Therapies Clinical Service Unit, Oxford University Hospitals NHS Foundation Trust, Oxford, United Kingdom

${ }^{5}$ Radcliffe Department of Medicine, University of Oxford, Oxford, United Kingdom

${ }^{6}$ Kadoorie Centre for Critical Care Research and Education, Oxford University Hospitals NHS Trust, Oxford, United Kingdom
}

\section{Corresponding Author:}

Carlos Morgado Areia, MSc, PT

Critical Care Research Group, Nuffield Department of Clinical Neurosciences

University of Oxford

Kadoorie Research Centre

John Radcliffe Hospital, Headley Way

Oxford, OX3 9DU

United Kingdom

Phone: 441865231440

Email: carlos.morgadoareia@ndcn.ox.ac.uk

\begin{abstract}
Background: The standard of care in general wards includes periodic manual measurements, with the data entered into track-and-trigger charts, either on paper or electronically. Wearable devices may support health care staff, improve patient safety, and promote early deterioration detection in the interval between periodic measurements. However, regulatory standards for ambulatory cardiac monitors estimating heart rate (HR) and respiratory rate (RR) do not specify performance criteria during patient movement or clinical conditions in which the patient's oxygen saturation varies. Therefore, further validation is required before clinical implementation and deployment of any wearable system that provides continuous vital sign measurements.

Objective: The objective of this study is to determine the agreement between a chest-worn patch (VitalPatch) and a gold standard reference device for HR and RR measurements during movement and gradual desaturation (modeling a hypoxic episode) in a controlled environment.

Methods: After the VitalPatch and gold standard devices (Philips MX450) were applied, participants performed different movements in seven consecutive stages: at rest, sit-to-stand, tapping, rubbing, drinking, turning pages, and using a tablet. Hypoxia was then induced, and the participants' oxygen saturation gradually reduced to $80 \%$ in a controlled environment. The primary outcome measure was accuracy, defined as the mean absolute error (MAE) of the VitalPatch estimates when compared with HR and RR gold standards (3-lead electrocardiography and capnography, respectively). We defined these as clinically acceptable if the rates were within 5 beats per minute for HR and 3 respirations per minute (rpm) for RR.

Results: Complete data sets were acquired for 29 participants. In the movement phase, the HR estimates were within prespecified limits for all movements. For RR, estimates were also within the acceptable range, with the exception of the sit-to-stand and turning page movements, showing an MAE of 3.05 (95\% CI 2.48-3.58) rpm and 3.45 (95\% CI 2.71-4.11) rpm, respectively. For the hypoxia phase, both HR and RR estimates were within limits, with an overall MAE of $0.72(95 \%$ CI $0.66-0.78)$ beats per minute and 1.89 (95\% CI 1.75-2.03) rpm, respectively. There were no significant differences in the accuracy of HR and RR estimations between normoxia ( $\geq 90 \%)$, mild ( $89.9 \%-85 \%)$, and severe hypoxia $(<85 \%)$.
\end{abstract}


Conclusions: The VitalPatch was highly accurate throughout both the movement and hypoxia phases of the study, except for RR estimation during the two types of movements. This study demonstrated that VitalPatch can be safely tested in clinical environments to support earlier detection of cardiorespiratory deterioration.

Trial Registration: ISRCTN Registry ISRCTN61535692; https://www.isrctn.com/ISRCTN61535692

(J Med Internet Res 2021;23(9):e27547) doi: 10.2196/27547

\section{KEYWORDS}

clinical validation; chest patch; vital signs; remote monitoring; wearable; heart rate; respiratory rate

\section{Introduction}

\section{Background}

Failure to recognize and act on physiological indicators of deterioration in hospital wards is a well-recognized problem [1-3]. Current practice involves manual measurement of vital signs and the use of early warning scores. However, the acquisition of these measurements can be time consuming for health professionals in a busy ward [4]; therefore, the desired monitoring frequency of observations is often not achieved [5]. This is identified as a limitation of periodic monitoring systems, as they are dependent on the frequency of vital sign observations [6]; of more concern is that even when the ideal frequency is achieved, patients might deteriorate between observation sets [7]. Continuous vital sign monitoring using wearable devices may overcome these limitations and improve timely detection of deterioration [8-10]. However, recent observational studies and pilot randomized controlled trials of wearable monitoring devices were inconclusive, and large randomized clinical trials of ambulatory monitoring devices are required to demonstrate improved patient outcomes $[1,8,11,12]$. This may be because of limitations in currently available ambulatory monitoring devices; for instance, it has been demonstrated that the detection and reduction of noise and artifacts during movement can be challenging in this type of monitoring [13]. Therefore, there is a need for diagnostic accuracy studies addressing motion artifacts to support the development of reliable wearable devices $[3,9,14,15]$.

The VitalPatch [16] is a wireless, battery-operated chest-worn wearable biosensor that records heart rate (HR), electrocardiography (ECG), HR variability, R-R interval (the time elapsed between two successive R-waves of the QRS signal on the electrocardiogram), respiratory rate (RR), body temperature, skin temperature, fall detection, activity (including step count), and posture (body position relative to gravity including fall detection) $[17,18]$. This device (Figure 1) was well tolerated by users because of its wearability and low impact on activities $[19,20]$. However, validation studies regarding the VitalPatch showed mixed results [3]; for HR, the mean bias was within acceptable ranges for all validation studies $[14,18,21-23]$ and within acceptable limits of agreement (LoA) in 3 studies [18,21,22]. For RR, the mean bias was also acceptable; however, all studies reported LoA outside of the clinically acceptable range [14,18,21-23].

Figure 1. Picture of a VitalPatch device.

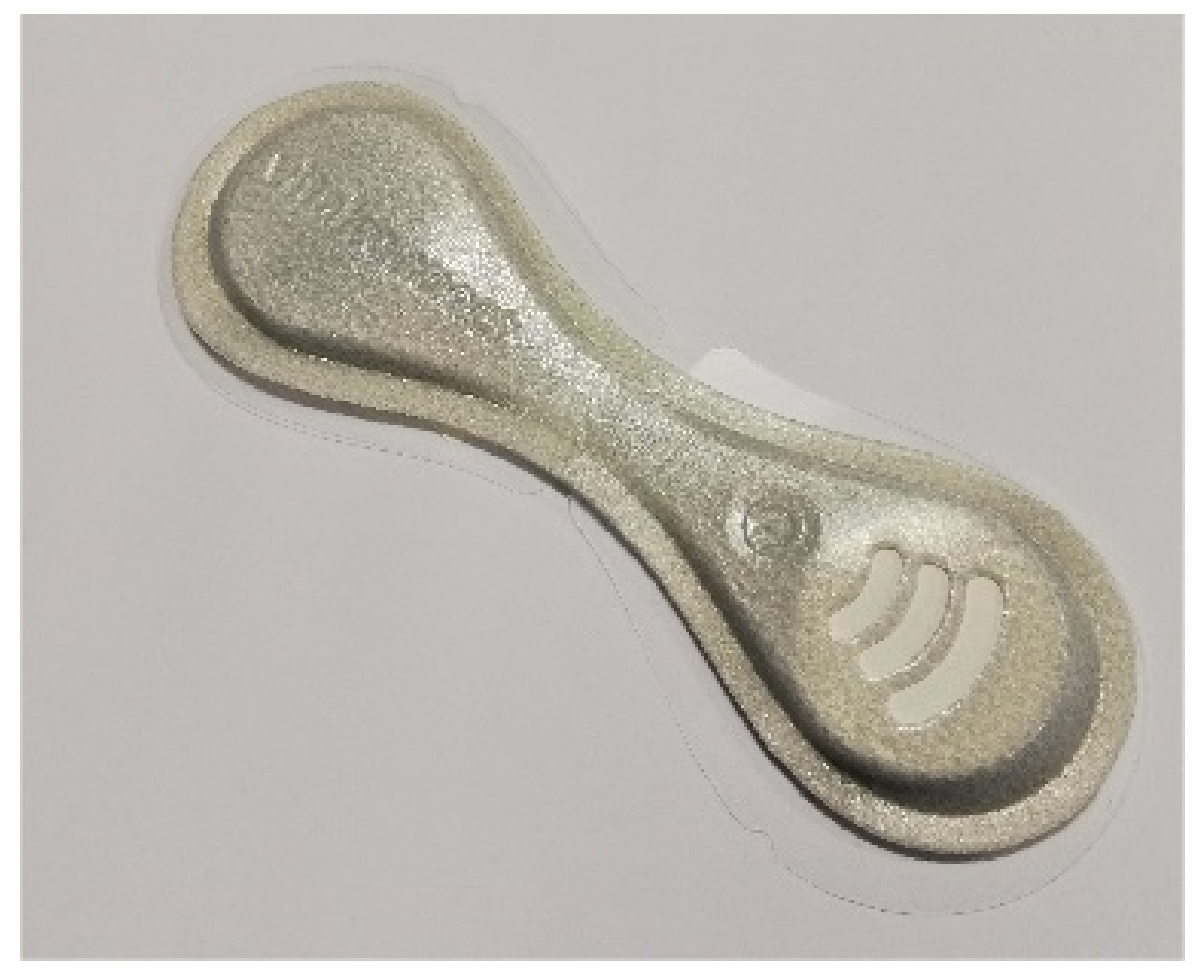


This work is a substudy of our virtual high dependency unit (vHDU) hypoxia study [24]. In the main protocol, our goal was to determine the specificity and sensitivity of currently available ambulatory pulse oximeters for the detection of hypoxemia. In this substudy, the aim is to assess the VitalPatch estimation accuracy during several simulated movements and in controlled hypoxic conditions against gold standard measurements, ECG for HR [25] and capnography for RR [26].

\section{Objectives}

The primary outcome is accuracy, defined as the mean absolute error (MAE) of the VitalPatch HR and RR estimation versus gold standard recording of HR (3-lead ECG) and RR (capnography) in healthy volunteers during movement and during induced hypoxia (down to peripheral oxygen saturation $\left[\mathrm{SpO}_{2}\right]$ of $\left.80 \%\right)$. In line with the American National Standards Institute/Association for the Advancement of Medical Instrumentation (ANSI/AAMI) EC13:2002 standard [27], we defined the MAE of the patch estimates to be clinically acceptable if the rates are within 5 beats per minute (bpm) and 3 respirations per minute (rpm) [3,21,22,27].

\section{Methods}

This manuscript follows the Guidelines for Reporting Reliability and Agreement Studies [28].

\section{Study Design}

This was a prospective, observational, cross-sectional cohort study that validated the HR and RR of a single-lead ECG chest patch. The VitalPatch HR is computed via the detection of $Q$ wave, $R$ wave, and $S$ wave complexes from a single-lead $E C G$ waveform. The RR is derived from combined information from three sources, that is, an embedded algorithm uses a weighted average of two characteristics of the ECG signal: (1) Q-, R-, and $\mathrm{S}$-wave amplitude modulation and (2) respiratory sinus arrhythmia (as both ECG-derived signals change during inspiration and expiration), and (3) changes in the accelerometer signal induced by chest movement during respiration $[23,29,30]$. We compared the HR and RR estimates with the gold standard HR derived from 3-lead ECG and RR determined from capnography measurements, respectively. The protocol for the full study has been published [24].

\section{Participants}

The screening process and inclusion and exclusion criteria are described in the study protocol [24]. Participants were recruited consecutively from the Oxford area, United Kingdom, between June 18 and August 8, 2019. The study sessions took place at the Cardiovascular Clinical Research Facility, John Radcliffe Hospital, Oxford, United Kingdom.

\section{Device Placement}

After the participants provided written informed consent, an arterial line was inserted into the participant's nondominant radial artery. The participants were then fitted with a VitalPatch in a modified lead-II configuration on the left midclavicular line over the intercostal space (as recommended by the vendor), a nonambulatory Philips MX 450 pulse oximeter (dominant arm), a 3-lead ECG (standard configuration) for acquisition of
$H R$, and a nasal cannula for acquisition of RR, both connected to the Philips monitor. The participants were continuously monitored with these devices throughout the study session by trained health care professionals with appropriate resuscitation facilities.

\section{Test Methods}

\section{Stage 1: Movement Phase}

The participants were considered at rest after fitting all the devices (variable duration between participants). The participants then moved into a chair and were asked to complete a series of consecutive motion conditions: $20 \times$ sit-to-stand (STS), 2-minute tapping at $2 \mathrm{~Hz}, 2$-minute rubbing at $2 \mathrm{~Hz}, 20$ times drinking from a plastic cup, 50 times turning pages, and a set of predefined table activity tasks [24]. STS was considered a dynamic activity, whereas the remainder were considered sedentary activities.

\section{Stage 2: Hypoxia Exposure Phase}

Following the movement phase, participants were placed in a semirecumbent, supine position and wore a tight-fitting silicone facemask connected to a hypoxicator unit (Everest Summit Hypoxic Generator). During this phase, oxygen saturations from the clinical standard Philips monitor guided the titration of the hypoxicator by a senior anesthetist from the research team. Oxygen (7\%) in nitrogen was used to further lower the fraction of inspired oxygen, if required [24]. The inhaled fraction of inspired oxygen was also monitored using an in-line gas analyzer. Participants were gradually desaturated to reach stable prespecified target $\mathrm{SpO}_{2}$ levels of $95 \%, 90 \%, 87 \%, 85 \%, 83 \%$, and $80 \%$. These levels were selected to allow sufficient data points for different hypoxia levels. $\mathrm{SpO}_{2}$ stability was subjective for each target window, that is, a senior anesthetist decided when a stable oxygen level was achieved.

\section{Data Collection}

For each participant, the following information was recorded in the case report form: age, sex, height, weight, skin type (Fitzpatrick scale [31]), baseline HR (Philips MX 450 3-lead ECG), and arterial oxygen saturation. Data from all participants were identified using a study number.

All data collection devices (wearables, tablets, laptops, and physiological monitors), and respective software, were synchronized to the same time server at the start of each study session day. Continuous HR and RR data (sampled at $0.25 \mathrm{~Hz}$ ) from the VitalPatch were sent via Bluetooth Low Energy to an Android tablet-based data capture system, developed in-house. Once the VitalPatch is connected to the tablet, it updates its internal clock and timestamps each sample internally. The Phillips MX 450 3-lead ECG data were collected in real time on a laptop using the ixTrend version 2.1 software (ixcellence $\mathrm{GmbH}$ ) [32]. For each step of the movement phase and prespecified oxygen saturation level of the hypoxia phase, the corresponding start and stop time stamps were recorded both in the in-house data capture software and on the respective case report forms [24]. 


\section{Data Analysis}

\section{Sample Size}

Our sample size calculation was based on recommendations from the International Organization for Standardization 80601-2-61:2019 (as the primary objective of the original study was the assessment of pulse oximeter accuracy). For our secondary analysis of the VitalPatch, we aimed to have approximately 30 complete patient data sets to ensure sufficient data points for analysis and validation testing using the VitalPatch and gold standard (Philips monitor) continuous data.

\section{Statistical Analysis}

To analyze the agreement between the VitalPatch and the gold standard during the movement phase, we compared 40-second median HR and RR estimates from the VitalPatch with simultaneous 40-second median HR and RR estimates from the Phillips monitor from each movement task interval $( \pm 20$ seconds at the start and at the end, respectively, to accommodate for the 40 -second window size). For the hypoxia phase (100\% to $80 \%$ saturations), 40-second median HR and RR estimates were also sampled for both devices from the start until the end of hypoxia testing. For each window, simultaneous 40-second median $\mathrm{SpO}_{2}$ estimates from the Philips monitor were also calculated per participant (our pulse oximetry hypoxia study suggested a negligible mean bias between the Philips $\mathrm{SpO}_{2}$ and arterial oxygen saturation). The 40-second window was chosen as the VitalPatch outputs instantaneous HR and RR estimates every 4 seconds, which gives 10 points per median estimate; the Philips monitor outputs both at $1 \mathrm{~Hz}$. Therefore, this represents the comparison of a 10-point median with a 40-point median centered on the same sample, which is reasonable.

For each phase, descriptive summaries for both devices are shown (number of data points and mean and SD values of HR and RR) and the MAE and root mean square error (RMSE) were computed for these vital signs. However, MAE was the metric used to determine HR and RR accuracy, in accordance with the ANSI/AAMI EC13:2002 standard [27]. It was defined as clinically acceptable if $\leq 5 \mathrm{bpm}$ and $3 \mathrm{rpm}$, respectively. Both MAE and RMSE 95\% CIs were determined via bootstrap using 10,000 replicates [33].

Bland-Altman plots, the corresponding mean bias, and the $95 \%$ LoA (SD 1.96) were also analyzed [34]. Finally, the Pearson correlation coefficient and associated $P$ value [35] were reported. All statistical tests were conducted using R v3.6.1 (R Foundation for Statistical Computing) [36].

\section{Missing Values}

Participants in whom it was not possible to achieve hypoxemia $\left(\mathrm{SpO}_{2}<90 \%\right)$ were excluded from the analysis to avoid different sample sizes or participants in the movement and hypoxia phases. All the included participants had complete data from the movement phase. If there were any technical issues during an activity, it was repeated until complete data were obtained. For clinical reasons, this was not possible in the hypoxic phase. Participants with intermittent missing data (if not significant) were included. We did not use imputation in this study, given that the median was calculated for consecutive 40-second windows without data on either the VitalPatch or reference device excluded from the analysis.

\section{Results}

\section{Participants}

The flow diagram of the data set collected in this study is shown in Figure 2. A total of 42 participants were recruited, of which $29(69 \%)$ were included in our analysis. The demographic descriptors are listed in Table 1. Exemplar HR and RR traces collected in the movement and hypoxia phases for one participant are shown below.

Figure 2. Participant flowchart. ABG: arterial blood gas.

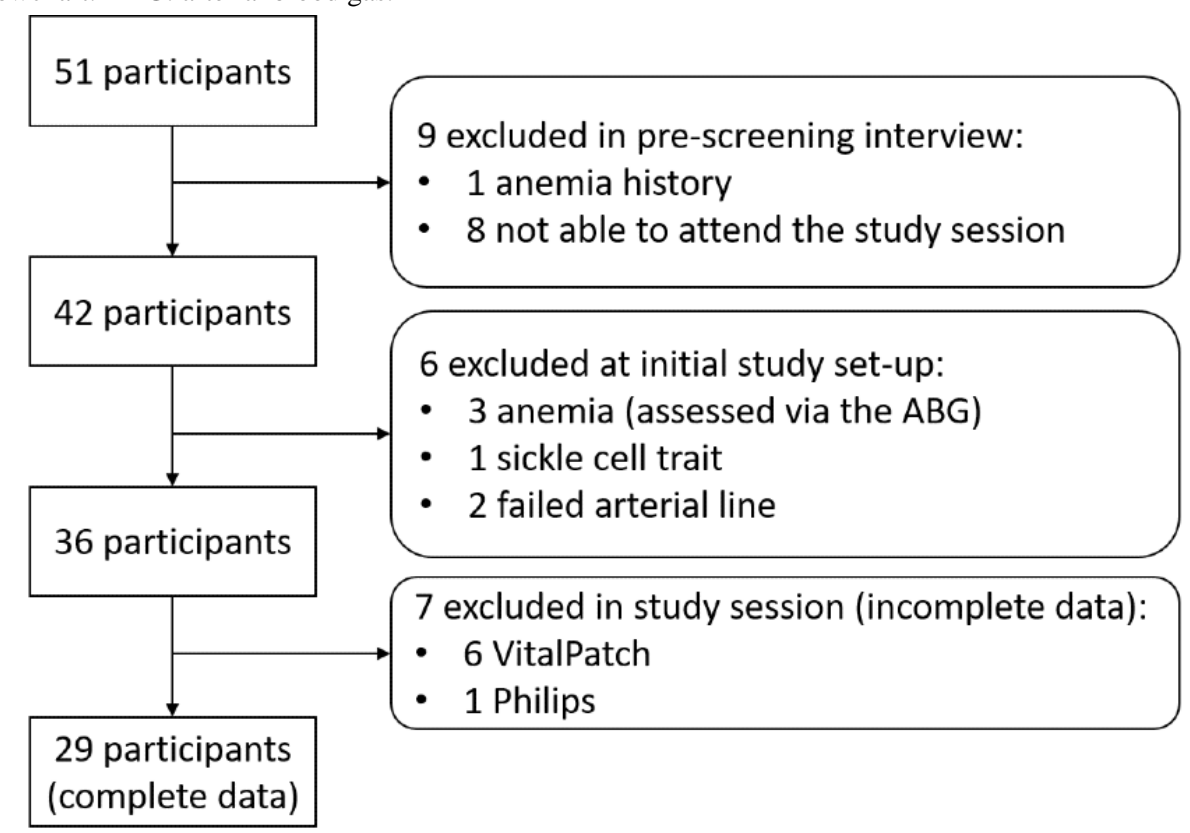


Table 1. Participants' demographics $(\mathrm{N}=29)$.

\begin{tabular}{|c|c|}
\hline Characteristics & Values \\
\hline \multicolumn{2}{|l|}{ Age (years) } \\
\hline Mean (SD) & $32.5(10.5)$ \\
\hline Median (IQR) & $29(25-38)$ \\
\hline Sex (female), n (\%) & $16(55)$ \\
\hline \multicolumn{2}{|l|}{ Height (m) } \\
\hline Mean (SD) & $1.7(0.1)$ \\
\hline Median (IQR) & $1.7(1.6-1.8)$ \\
\hline \multicolumn{2}{|l|}{ Weight (kg) } \\
\hline Mean (SD) & $70.6(14.1)$ \\
\hline Median (IQR) & $68(59.2-83)$ \\
\hline \multicolumn{2}{|l|}{ BMI $\left(\mathrm{kg} / \mathrm{m}^{2}\right)$} \\
\hline Mean (SD) & $24.3(3.9)$ \\
\hline Median (IQR) & $24(21.5-26.8)$ \\
\hline \multicolumn{2}{|l|}{ Skin tone, $\mathrm{n}(\%)$} \\
\hline Type 1 & $7(24)$ \\
\hline Type 2 & $12(41)$ \\
\hline Type 3 & $2(7)$ \\
\hline Type 4 & $8(28)$ \\
\hline \multicolumn{2}{|l|}{ Respiratory rate $\left(\mathrm{rpm}^{\mathrm{a}}\right)$} \\
\hline Mean (SD) & $16.3(3.6)$ \\
\hline Median (IQR) & $17(13-19)$ \\
\hline \multicolumn{2}{|l|}{ Heart rate $\left(\mathbf{b p m}^{\mathbf{b}}\right)$} \\
\hline Value, mean (SD) & $72.9(11.9)$ \\
\hline Value, median (IQR) & $73(64-82)$ \\
\hline \multicolumn{2}{|l|}{$\mathrm{SaO}_{2}{ }^{\mathrm{c}}$} \\
\hline Mean (SD) & $99.3(1.3)$ \\
\hline Median (IQR) & $100(99-100)$ \\
\hline \multicolumn{2}{|l|}{ Systolic BP $\mathbf{P}^{\mathbf{d}}(\mathrm{mm} \mathrm{Hg})$} \\
\hline Mean (SD) & $134.1(20.0)$ \\
\hline Median (IQR) & $129(123-146)$ \\
\hline \multicolumn{2}{|l|}{ Diastolic BP (mm Hg) } \\
\hline Mean (SD) & $78.5(11.2)$ \\
\hline Median (IQR) & $75(70-87)$ \\
\hline
\end{tabular}

${ }^{\mathrm{a}} \mathrm{rpm}$ : respirations per minute.

$\mathrm{b}_{\mathrm{bpm}}$ : beats per minute.

${ }^{\mathrm{c}} \mathrm{SaO}_{2}$ : arterial oxygen saturation.

${ }^{\mathrm{d}}$ BP: blood pressure.

\section{Movement Phase}

The movement phase lasted approximately 13 minutes for each participant. The exemplar HR and RR traces shown in Figure 3 confirm higher variability during the STS movement. The scatter and Bland-Altman plots for HR and RR in Figure 4 also show a higher mean bias and LoA for the dynamic activity (STS) versus one of the sitting movements (using a tablet). Scatter and Bland-Altman plots for all movement tests can be found in Multimedia Appendix 1. Table 2 lists the performance 
metrics for the complete movement phase. The VitalPatch HR showed marginal overestimation throughout the sitting activities, with a mean MAE ranging from 0.55-0.84 bpm, RMSE from $0.89-1.32 \mathrm{bpm}$, bias from $0.06-0.32 \mathrm{bpm}$, and with a correlation of approximately $1(P<.001)$ throughout. In the dynamic activity
(STS), there was an increase in the MAE, 2.69 (95\% CI 1.58-3.63) bpm, and RMSE 5.75 (95\% CI 3.83-7.94) bpm, mean bias 1.92 (95\% LoA -8.75 to 12.59$) \mathrm{bpm}$, with a correlation of approximately $0.95(P<.001)$.

Figure 3. (A) Exemplar heart rate and (B) respiratory rate data collected during the movement phase for one participant (vHDU-H-029). Red: VitalPatch heart rate; blue: VitalPatch respiratory rate; black: Philips 3-Lead electrocardiography heart rate in part (A) and capnography respiratory rate in part (B); brown horizontal line: movement tests. bpm: beats per minute; HR: heart rate; rpm: respirations per minute; RR: respiratory rate; STS: sit-to-stand.

\section{Movement phase}

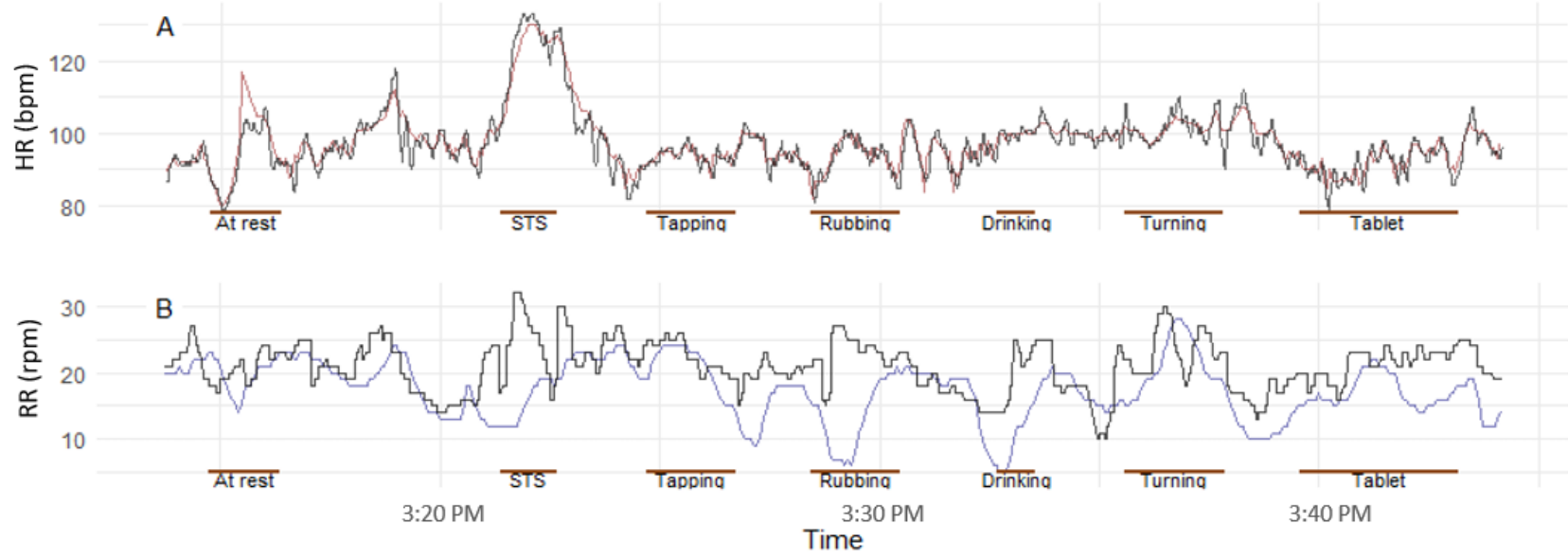

Figure 4. (A and C) Scatter plots and (B and D) Bland-Altman plots for heart rate (A and B in red) and respiratory rate (C and D in blue) in two example movements: (1) repetitive sit-to-stand and (2) using a tablet (while sitting). bpm: beats per minute; HR: heart rate; rpm: respirations per minute; RR: respiratory rate.

HR: Tablet

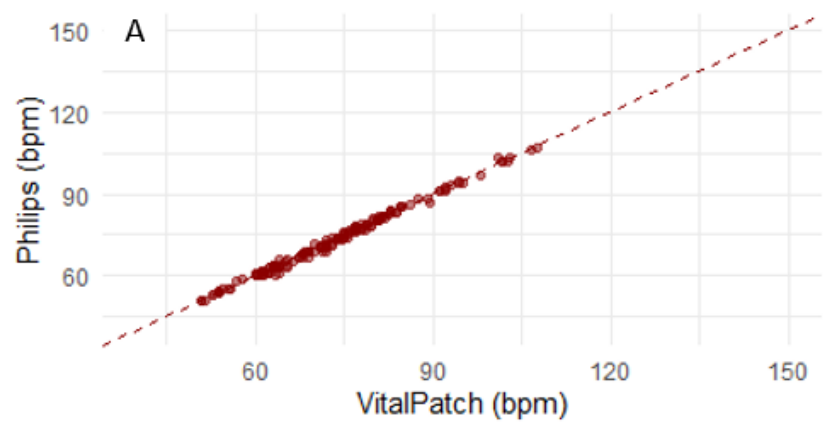

B

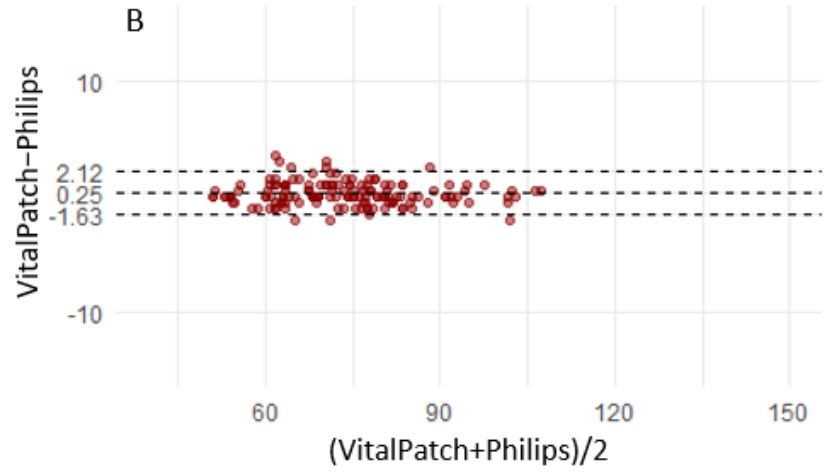

RR: Tablet

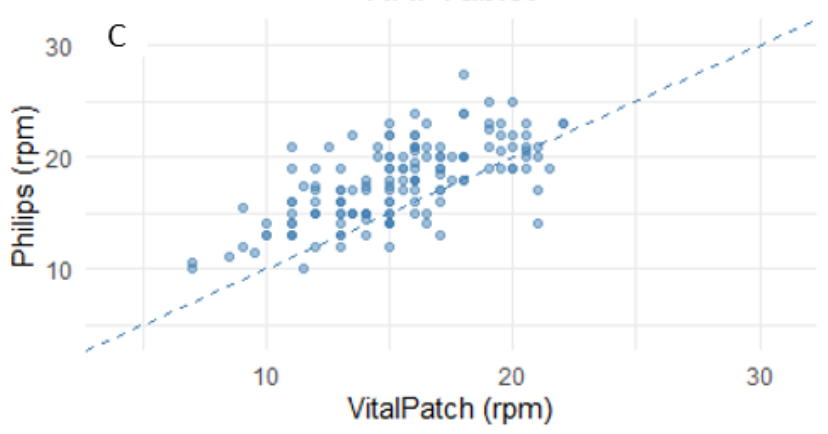

D

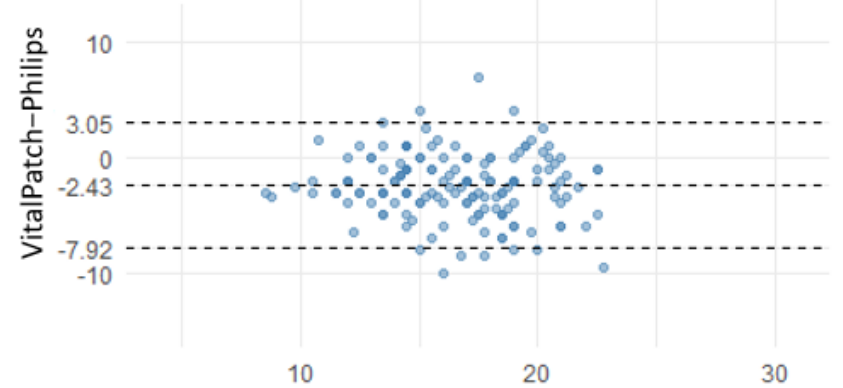

(VitalPatch+Philips)/2 
Table 2. Movement phase metrics ( $\mathrm{N}=29)$.

\begin{tabular}{|c|c|c|c|c|c|c|c|}
\hline Movement & $\begin{array}{l}\text { Number of } \\
\text { data points }\end{array}$ & $\begin{array}{l}\text { Philips, mean } \\
\text { (SD) }\end{array}$ & $\begin{array}{l}\text { VitalPatch, } \\
\text { mean (SD) }\end{array}$ & $\begin{array}{l}\text { Pearson corre- } \\
\text { lation }\left(\mathrm{R}^{2}\right)^{\mathrm{a}}\end{array}$ & $\operatorname{MAE}^{\mathrm{b}}(95 \% \mathrm{CI})$ & $\operatorname{RMSE}^{\mathrm{c}}(95 \% \mathrm{CI})$ & $\begin{array}{l}\text { Mean bias }(95 \% \\
\left.\text { LoA }^{d}\right)\end{array}$ \\
\hline \multicolumn{8}{|c|}{ Heart rate $\left(b_{p m}{ }^{e}\right)$} \\
\hline At rest & 216 & $71.87(12.52)$ & $72.18(12.86)$ & 0.99 & 1.16 (0.91 to 1.39$)$ & 2.11 (1.62 to 2.62$)$ & $0.31(-3.8$ to 4.42$)$ \\
\hline $\operatorname{STS}^{\mathrm{f}}$ & 93 & $89.94(17.32)$ & $91.87(16.38)$ & 0.95 & 2.69 (1.58 to 3.63$)$ & 5.75 (3.83 to 7.94$)$ & $1.92(-8.75$ to 12.59$)$ \\
\hline Tapping & 111 & 70.99 (13.89) & $71.07(14.10)$ & 1 & 0.55 (0.42 to 0.68$)$ & 0.89 (0.66 to 1.12$)$ & 0.08 ( -1.68 to 1.83$)$ \\
\hline Rubbing & 107 & $71.90(12.69)$ & 72.06 (12.69) & 1 & 0.69 (0.51 to 0.85$)$ & $1.12(0.88$ to 1.37$)$ & 0.15 (-2.03 to 2.34$)$ \\
\hline Drinking & 76 & $71.93(13.91)$ & $72.25(13.68)$ & 1 & 0.84 (0.61 to 1.05$)$ & 1.32 (0.98 to 1.67$)$ & $0.32(-2.21$ to 2.84$)$ \\
\hline $\begin{array}{l}\text { Turning } \\
\text { pages }\end{array}$ & 98 & $75.48(13.71)$ & $75.54(13.58)$ & 1 & $0.72(0.52$ to 0.90$)$ & 1.21 (0.80 to 1.57$)$ & 0.06 ( -2.32 to 2.44$)$ \\
\hline Tablet & 152 & $73.18(12.45)$ & $73.43(12.38)$ & 1 & 0.69 (0.58 to 0.80$)$ & $0.99(0.85$ to 1.13$)$ & 0.25 ( -1.63 to 2.12$)$ \\
\hline \multicolumn{8}{|c|}{ Respiratory rate $\left(\mathbf{r p m}^{\mathbf{g}}\right)$} \\
\hline At rest & 127 & $17.26(3.29)$ & $15.31(3.12)$ & 0.72 & 2.44 (2.11 to 2.77$)$ & 3.10 (2.70 to 3.48$)$ & -1.94 (-6.68 to 2.79$)$ \\
\hline STS & 92 & $18.41(4.71)$ & $16.83(5.20)$ & 0.56 & $3.45(2.71$ to 4.11$)$ & 4.89 (3.91 to 5.92$)$ & $-1.59(-10.7$ to 7.53$)$ \\
\hline Tapping & 104 & $18.43(2.75)$ & $16.75(3.06)$ & 0.68 & 2.14 (1.76 to 2.50$)$ & $2.86(2.40$ to 3.35$)$ & $-1.68(-6.25$ to 2.89$)$ \\
\hline Rubbing & 103 & $17.50(3.51)$ & $15.52(3.13)$ & 0.63 & $2.44(1.93$ to 2.88$)$ & $3.48(2.45$ to 4.38$)$ & $-1.97(-7.63$ to 3.69$)$ \\
\hline Drinking & 72 & $16.90(3.76)$ & $15.46(3.48)$ & 0.74 & 2.30 (1.83 to 2.72$)$ & 2.99 (2.36 to 3.62$)$ & $-1.44(-6.62$ to 3.75$)$ \\
\hline $\begin{array}{l}\text { Turning } \\
\text { pages }\end{array}$ & 97 & $19.09(4.08)$ & $17.41(3.72)$ & 0.53 & $3.05(2.48$ to 3.58$)$ & $4.13(3.32$ to 4.90$)$ & $-1.69(-9.11$ to 5.73$)$ \\
\hline Tablet & 152 & $17.75(3.51)$ & $15.32(3.23)$ & 0.66 & 2.97 (2.61 to 3.31$)$ & $3.70(3.31$ to 4.10$)$ & -2.43 (-7.92 to 3.05$)$ \\
\hline
\end{tabular}

${ }^{\mathrm{a}} P<.001$.

${ }^{b}$ MAE: mean absolute error.

${ }^{\mathrm{c}} \mathrm{RMSE}$ : root mean square error.

${ }^{\mathrm{d}}$ LoA: limits of agreement.

ebpm: beats per minute.

${ }_{\text {f }}$ TS: sit-to-stand.

$\mathrm{g}_{\text {rpm: respirations per minute. }}$

In contrast, a consistent underestimation was observed for RR throughout the sitting activities. MAE ranged from 2.14-3.05 rpm, RMSE from 2.86-4.13, mean bias from -1.68 to -2.43 rpm, and the correlation coefficient from 0.53-0.74 $(P<.001)$. During the dynamic activity, the MAE was 3.45 (95\% CI
2.71-4.11) rpm, RMSE was 4.89 (95\% CI 3.91-5.92) rpm, mean bias was $-1.59(95 \%$ LoA -10.7 to 7.53$) \mathrm{rpm}$, and the correlation was $0.56(P<.001)$. An overview of the MAE, RMSE, and mean bias (LoA) for all movements is shown in Figure 5. 
Figure 5. Accuracy and Bias (mean bias) plots for all movement tests. Red: heart rate; horizontal red line represents acceptable limits. Blue: respiratory rate; horizontal blue line represents acceptable limits. (A) MAE (95\% CI) plot, (B) RMSE (95\% CI) plot, (C) bias LOAs. bpm: beats per minute; LoAs: limits of agreement; MAE: mean absolute error; RMSE: root mean square error; rpm: respirations per minute; STS: sit-to-stand.

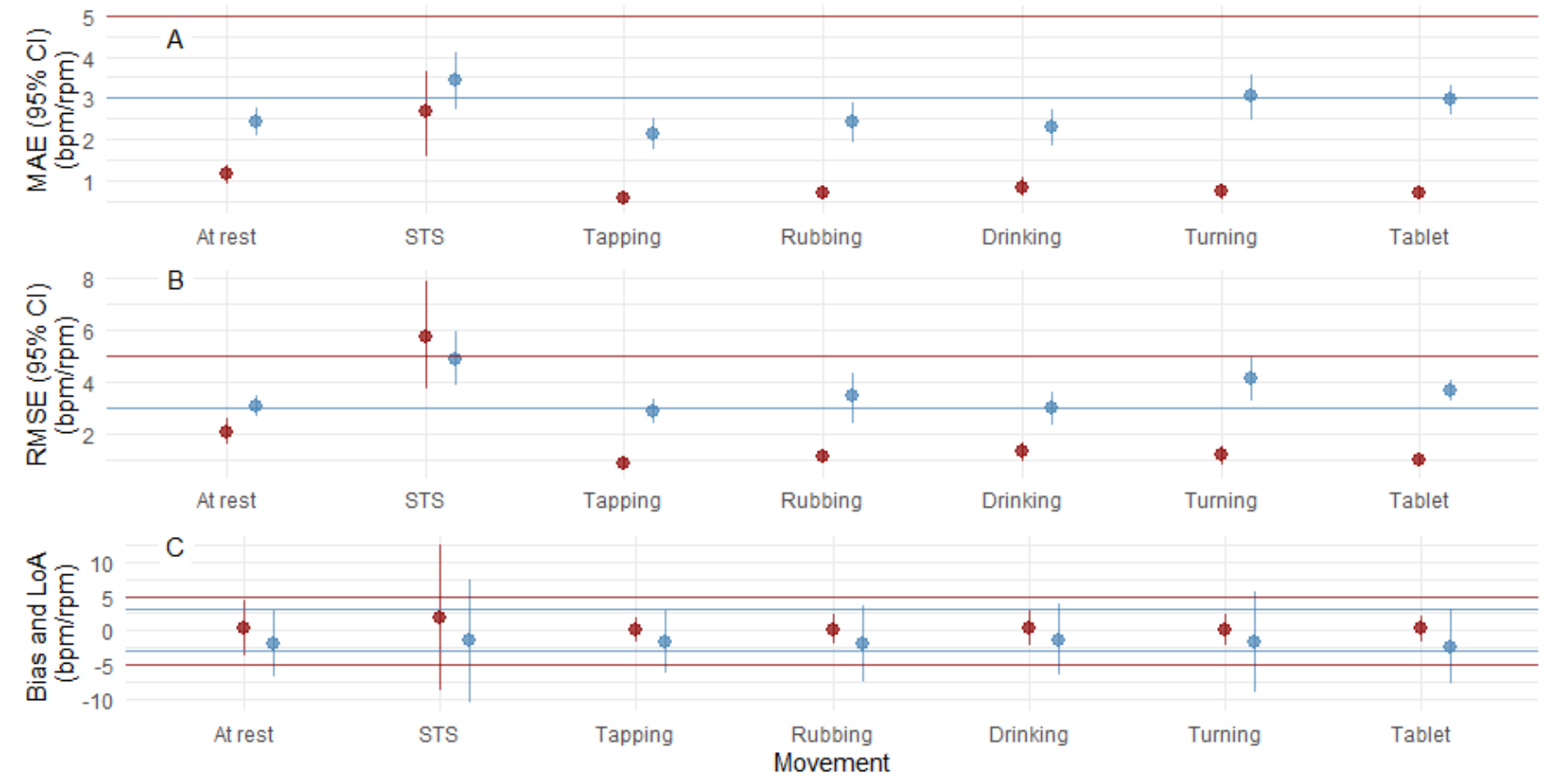

The VitalPatch HR estimation was within clinically acceptable accuracy, achieving a MAE 0.72 (95\% CI 0.66-0.78) bpm and a RMSE 1.16 (95\% CI 1.02-1.29) bpm in the hypoxia phase. Figure 6 shows an exemplar participant during the hypoxia phase, and Figure 7 shows the scatter and Bland-Altman plots for HR and RR. The mean bias was $0.49(95 \% \mathrm{LoA}-1.58$ to

$2.56)$, and the correlation was approximately $1 \quad(P<.001)$. Although more variable, the RR was also within the acceptable accuracy achieving a MAE 1.89 (95\% CI 1.75-2.03) rpm and a RMSE 2.92 (95\% CI 2.64-3.2) rpm. A minimal mean bias of -0.08 (95\% LoA -5.8 to 5.65) and a correlation of approximately $0.72(P<.001)$ were observed.

Figure 6. Exemplar heart rate (A) and respiratory rate (B) traces during the hypoxia phase for one participant (vHDU-H-29). red: VitalPatch heart rate; blue: VitalPatch respiratory rate; black: Philips 3-Lead electrocardiography heart rate (A) and capnography respiratory rate (B); brown horizontal line: target hypoxia levels. bpm: beats per minute; HR: heart rate; rpm: respirations per minute; RR: respiratory rate.

Hypoxia phase

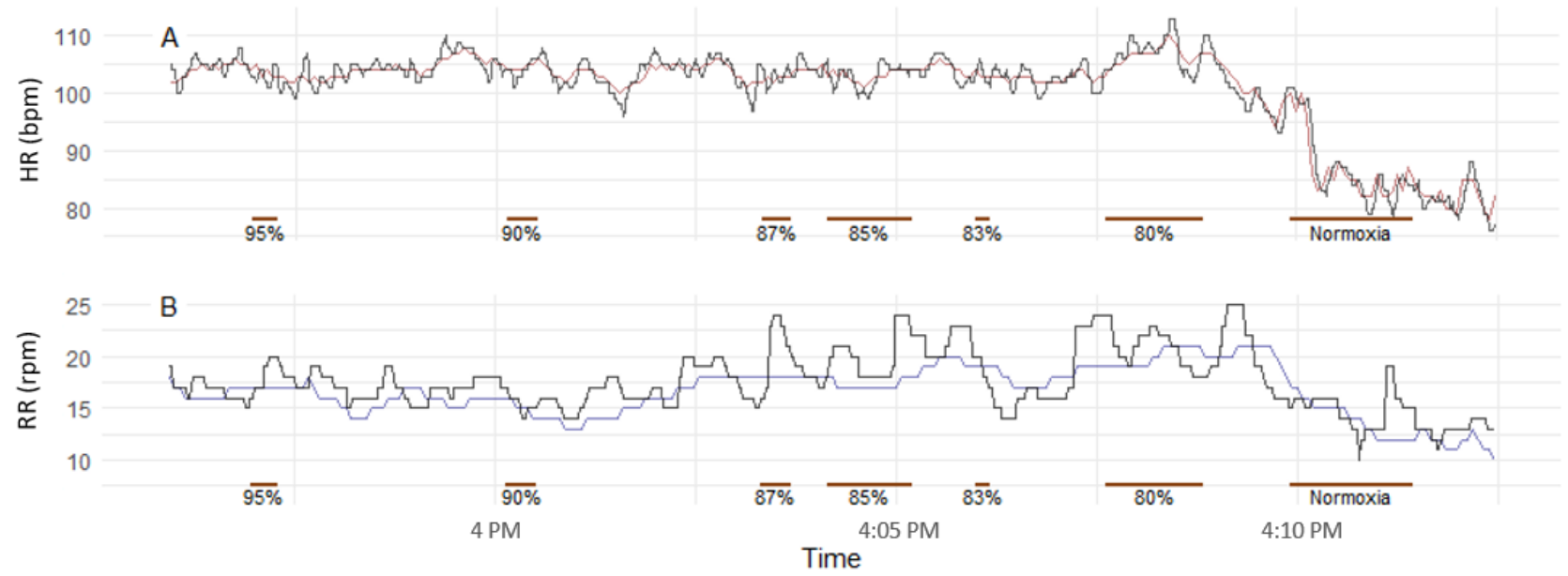


Figure 7. (A and C) Scatter plots and (B and D) Bland-Altman plots for heart rate (A and B in red) and respiratory rate (C and D in blue) during the full hypoxia phase. bpm: beats per minute; rpm: respirations per minute; $\mathrm{SpO}_{2}$ : peripheral oxygen saturation.

Hypoxia phase
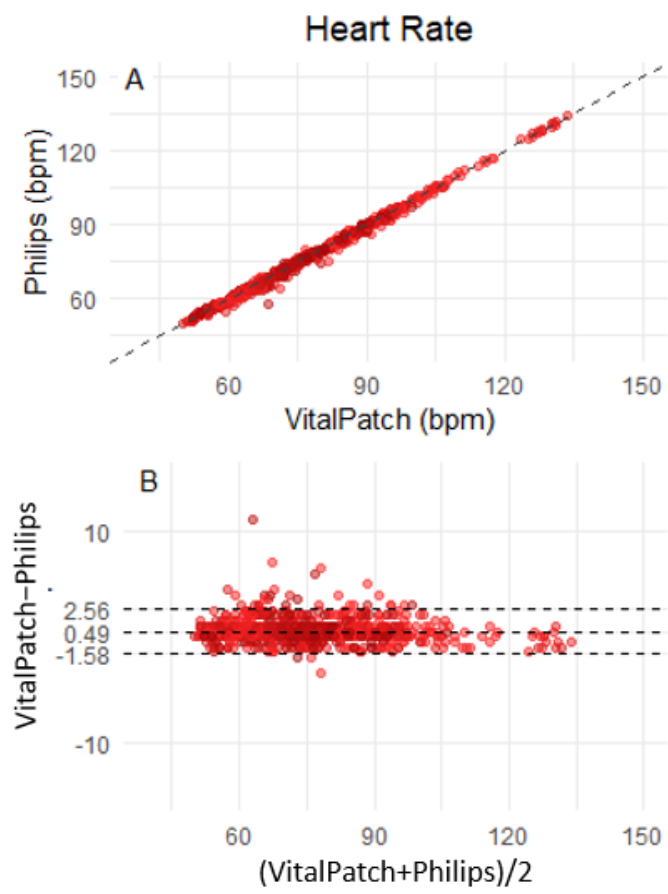

There were also no significant differences in the error metrics between the $\mathrm{SpO}_{2}$ subgroups (normoxia, $\mathrm{SpO}_{2} 100 \%$ to $90 \%$; mild hypoxia, $\mathrm{SpO}_{2} 85 \%-89.9 \%$; and severe hypoxia,
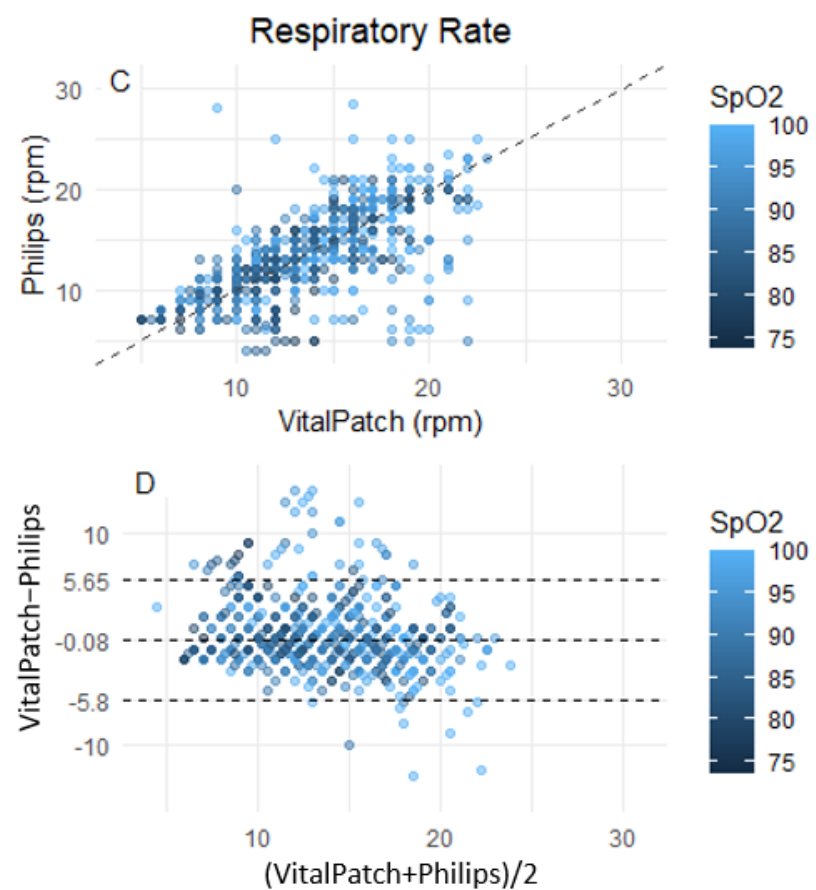

$\mathrm{SpO}_{2}<85 \%$ [24]), as shown in Multimedia Appendix 2. Finally, Figure 8 shows the MAE, RMSE, and mean bias (LoA) computed for each oxygen saturation level from the hypoxia phase.

Figure 8. Accuracy and Bias (mean bias) plots for the hypoxia phase. Red: heart rate; horizontal red lines represent acceptable limits Blue: respiratory rate; horizontal blue line represents acceptable limits. (A) MAE (95\% CI) plot, (B) RMSE (95\% CI) plot, (C) bias LOAs. bpm: beats per minute; LoA:s limits of agreement; MAE: mean absolute error; RMSE: root mean square error; rpm: respirations per minute; $\mathrm{SpO}_{2}$ : peripheral oxygen saturation.
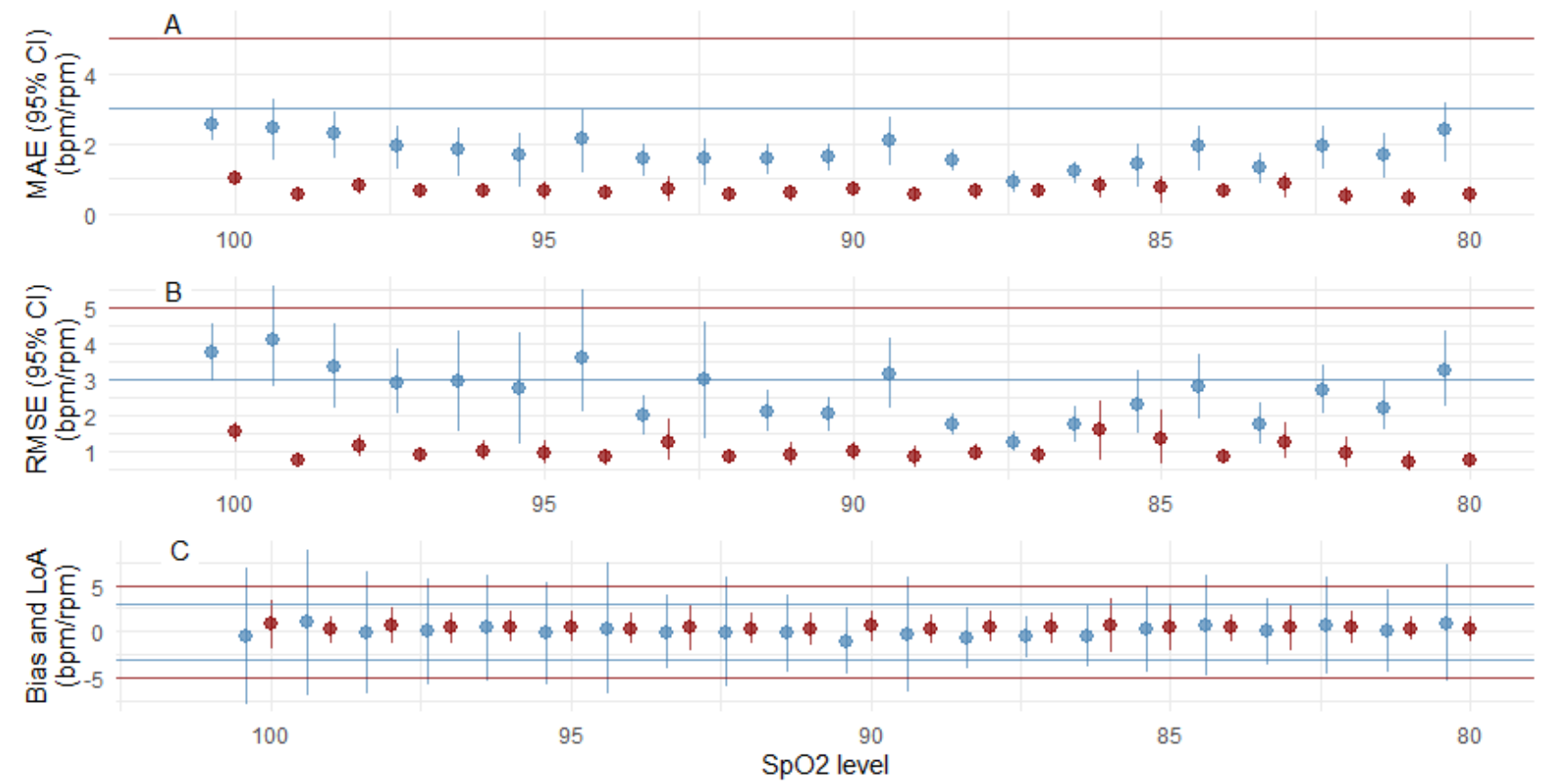


\section{Discussion}

\section{Principal Findings}

The key findings from this study were as follows: (1) The VitalPatch HR estimation was well within acceptable accuracy limits when compared with the gold standard device (Philips 3-lead ECG), (2) The VitalPatch RR estimation was also in agreement with the gold standard device (Philips capnography) for most of the movements and throughout the full hypoxia phase, and (3) there was no impact on the HR and RR estimation performance when participants were exposed to induced hypoxia. These findings support the clinical use of VitalPatch in patients with reduced baseline saturation or at risk of hypoxia.

Accuracy testing during motion is important before clinical use, as we aim to implement an ambulatory monitoring system that promotes patient mobility and independence without compromising safety. Our results suggest that the VitalPatch can measure HR with high accuracy and minimal bias during simple movements (mimicking in-hospital patient movements). Reliability was reduced during STS activity but was still within acceptable limits. These results agree with the results of previous clinical validation studies when compared with a reference standard device in healthy volunteers $[18,23]$ and surgical patients [21,22]. When compared with nurse manual measurements in surgical patients [14], VitalPatch meets the accuracy criteria for HR estimation.

The MAE of the VitalPatch RR estimation was comparatively higher, especially during the movement phase. However, it was also mostly within the prespecified limits (except for the STS and turning a page movements), in agreement with previous studies [14,18,21-23]. High variation during STS was expected as it is a dynamic activity, potentially creating several artifacts that could affect VitalPatch accuracy during this test [21,22]; however, a high MAE for RR was not expected for the turning pages activity (even if close to the 3-rpm threshold). We postulate that the latter may have created a low-frequency periodic artifact that influenced both the ECG and accelerometer waveforms (both used in the computation of the RR estimate by the VitalPatch).

The most recent study showed that the VitalPatch tended to overestimate RR with a bias of $4.4 \mathrm{rpm}$ [22], but previous studies showed a predominant RR underestimation [14,18,21]. Our study seems to agree with the latter, as VitalPatch mostly underestimated RR throughout the movement phase and varied in the hypoxia phase. However, in contrast to these previous studies, we collected vital signs from healthy participants.

This study also assessed the VitalPatch reliability during a controlled hypoxia phase, and our results suggest that there is no impact on this patch's reliability for HR and RR measurements during periods of reduced $\mathrm{SpO}_{2}$ levels. The VitalPatch estimates followed the gold standard HR and RR variations caused by the controlled desaturation of the participants during the hypoxia phase, reliably, supporting its use in clinical populations with reduced baseline saturation or at risk of hypoxia.

\section{Limitations}

This study had some limitations. First, the participants were healthy volunteers in a controlled environment that is not representative of hospitalized patients. In addition, the main study was powered to assess the accuracy of $\mathrm{SpO}_{2}$ and not that of HR and RR. Although the range required to validate RR was achieved (10 to $30 \mathrm{rpm}$ is required [18] and 4 to $31 \mathrm{rpm}$ was collected), the HR range achieved in this substudy was 40 to $150 \mathrm{bpm}$, a subset of the range required to validate its accuracy, 30 to $200 \mathrm{bpm}$ [27].

\section{Comparison With Prior Work and Future Research}

The ANSI/AAMI EC13:2002 standard [27] only requires that a measure of systematic error (eg, mean bias or MAE) be below the clinically accepted limits. In more recent studies, MAE has been used because it is less susceptible to outliers than the mean bias $[18,23,30]$. Analyzing the RMSE results, which combines both the systematic and random errors, we note that they follow the trend of those of the MAE metric as the RMSE was also higher during the STS activity for both variables and for the turning pages movement for RR. In our study, the LoA (also a measure of random error) seemed to be within the acceptable range when compared with the results of other studies [14,18,21-23] (except for the aforementioned cases). Previous studies reported higher LoA (defined as out of the acceptable range by the authors) for RR when compared with reference standard devices both in healthy volunteers ( -10.1 to 6.5$)$ [18] and surgical patients ( -15.8 to 11.2$)$ [21] and ( -4.4 to 13.3 ) [22], and when compared with nurse manual measurements in surgical patients (10.32 to 9.04) [14].

In this study, the VitalPatch was subjected to robust testing, during movement and under induced hypoxia, against a gold standard in a controlled environment. Our results suggest that this device can be safely tested in clinical environments (within the collected input range for each vital sign). Our results will inform future phases of our vHDU research project, aiming to develop an accurate wearable system for monitoring ambulatory patients in hospitals.

\section{Conclusions}

In conclusion, the VitalPatch was highly accurate throughout movement tests except for the measurement of RR during the STS activity and the turning pages movement. This device was also reliable throughout the hypoxia phase, with no significant MAE or bias differences in hypoxic levels. This study demonstrated that VitalPatch can be safely tested in clinical environments to support earlier detection of clinical deterioration.

\section{Acknowledgments}

CMA, MS, LY, MH, and SV were funded by the National Institute for Health Research (NIHR) Oxford Biomedical Research Centre. The views expressed are those of the authors and not necessarily those of the National Health Service, the NIHR, or the 
Department of Health. AS is currently supported by the NIHR Doctoral Research Fellowship (NIHR-DRF-2017-10-094). The authors would like to thank all the volunteers who participated in this study.

\section{Authors' Contributions}

Authorship was determined in accordance with the International Committee of Medical Journal Editors guidelines. CMA, SV, PP, LT, and PW drafted the initial protocol. CMA, SV, EK, JE, LY, OG, CR, MP, and MS conducted the study procedures and data acquisition. AS and MH provided medical coverage on the study days. CMA and MS conducted statistical analyses. CMA drafted the manuscript, and all the authors reviewed and approved the manuscript. The funders had no role in the study protocol design or in the collection, management, analysis, and interpretation of the data or the writing of the final report.

\section{Conflicts of Interest}

PW and LT report significant grants from the NIHR, United Kingdom, and the NIHR Biomedical Research Centre, Oxford, during the conduct of the study. PW and LT report modest grants and personal fees from Sensyne Health outside the submitted work. LT work part-time for Sensyne Health and hold shares in the company. PW was previously Chief Medical officer for Sensyne Health and has shares in the company. Sensyne Health have provided research support to his university department.

\section{Multimedia Appendix 1}

Scatter and Bland-Altman plots for movement phase.

[DOCX File, $160 \mathrm{~KB}$-Multimedia Appendix 1]

\section{Multimedia Appendix 2}

Hypoxia phase subgroups analysis.

[DOCX File, 176 KB-Multimedia Appendix 2]

\section{References}

1. Sun L, Joshi M, Khan S, Ashrafian H, Darzi A. Clinical impact of multi-parameter continuous non-invasive monitoring in hospital wards: a systematic review and meta-analysis. J R Soc Med 2020 Jun;113(6):217-224 [FREE Full text] [doi: 10.1177/0141076820925436] [Medline: 32521195]

2. McQuillan P, Pilkington S, Allan A, Taylor B, Short A, Morgan G, et al. Confidential inquiry into quality of care before admission to intensive care. Br Med J 1998 Jun 20;316(7148):1853-1858 [FREE Full text] [doi: 10.1136/bmj.316.7148.1853] [Medline: 9632403]

3. Leenen J, Leerentveld C, van Dijk JD, van Westreenen HL, Schoonhoven L, Patijn G. Current evidence for continuous vital signs monitoring by wearable wireless devices in hospitalized adults: systematic review. J Med Internet Res 2020 Jun 17;22(6):e18636 [FREE Full text] [doi: 10.2196/18636] [Medline: 32469323]

4. Downey CL, Tahir W, Randell R, Brown JM, Jayne DG. Strengths and limitations of early warning scores: a systematic review and narrative synthesis. Int J Nurs Stud 2017 Nov;76:106-119. [doi: 10.1016/j.ijnurstu.2017.09.003] [Medline: 28950188]

5. Clifton DA, Clifton L, Sandu D, Smith GB, Tarassenko L, Vollam SA, et al. 'Errors' and omissions in paper-based early warning scores: the association with changes in vital signs--a database analysis. BMJ Open 2015 Jul 03;5(7):e007376 [FREE Full text] [doi: 10.1136/bmjopen-2014-007376] [Medline: 26141302]

6. Jansen JO, Cuthbertson BH. Detecting critical illness outside the ICU: the role of track and trigger systems. Curr Opin Crit Care 2010 Jun;16(3):184-190. [doi: 10.1097/MCC.0b013e328338844e] [Medline: 20305556]

7. Tarassenko L, Hann A, Young D. Integrated monitoring and analysis for early warning of patient deterioration. Br J Anaesth 2006 Jul;97(1):64-68 [FREE Full text] [doi: 10.1093/bja/ael113] [Medline: 16707529]

8. Downey C, Randell R, Brown J, Jayne DG. Continuous versus intermittent vital signs monitoring using a wearable, wireless patch in patients admitted to surgical wards: pilot cluster randomized controlled trial. J Med Internet Res 2018 Dec 11;20(12):e10802 [FREE Full text] [doi: 10.2196/10802] [Medline: 30538086]

9. Joshi M, Ashrafian H, Aufegger L, Khan S, Arora S, Cooke G, et al. Wearable sensors to improve detection of patient deterioration. Expert Rev Med Devices 2019 Feb;16(2):145-154. [doi: 10.1080/17434440.2019.1563480] [Medline: 30580650]

10. Watkins T, Whisman L, Booker P. Nursing assessment of continuous vital sign surveillance to improve patient safety on the medical/surgical unit. J Clin Nurs 2016 Jan;25(1-2):278-281 [FREE Full text] [doi: 10.1111/jocn.13102] [Medline: 26531215]

11. Weller RS, Foard KL, Harwood TN. Evaluation of a wireless, portable, wearable multi-parameter vital signs monitor in hospitalized neurological and neurosurgical patients. J Clin Monit Comput 2018 Oct;32(5):945-951. [doi: 10.1007/s10877-017-0085-0] [Medline: 29214598] 
12. Paul JE, Chong MA, Buckley N, Harsha P, Shanthanna H, Tidy A, et al. Vital sign monitoring with continuous pulse oximetry and wireless clinical notification after surgery (the VIGILANCE pilot study)-a randomized controlled pilot trial. Pilot Feasibility Stud 2019 Feb 26;5:36 [FREE Full text] [doi: 10.1186/s40814-019-0415-8] [Medline: $\underline{30858986}$ ]

13. Hamilton P, Curley M, Aimi R, Sae-Hau C. Comparison of methods for adaptive removal of motion artifact. In: Proceedings of the Computers in Cardiology Conference. 2000 Presented at: Computers in Cardiology 2000; Sep 24-27, 2000; Cambridge, MA, USA. [doi: 10.1109/cic.2000.898537]

14. Weenk M, van Goor H, Frietman B, Engelen L, van Laarhoven CJ, Smit J, et al. Continuous monitoring of vital signs using wearable devices on the general ward: pilot study. JMIR Mhealth Uhealth 2017 Jul 05;5(7):e91 [FREE Full text] [doi: 10.2196/mhealth.7208] [Medline: 28679490]

15. Verrillo S, Cvach M, Hudson K, Winters B. Using continuous vital sign monitoring to detect early deterioration in adult postoperative inpatients. J Nurs Care Qual 2019;34(2):107-113. [doi: 10.1097/NCQ.0000000000000350] [Medline: $\underline{30095509]}$

16. VitalPatch. VitalConnect. URL: https://vitalconnect.com/solutions/vitalpatch/ [accessed 2021-08-09]

17. Instructions for use, VitalPatch ${ }^{\circledR}$ Biosensor. VitalConnect. URL: https://vitalconnect.com/docs/ifu006/revS/IFU-06 [accessed 2021-08-09]

18. Selvaraj N, Nallathambi G, Moghadam R, Aga A. Fully disposable wireless patch sensor for continuous remote patient monitoring. Annu Int Conf IEEE Eng Med Biol Soc 2018 Jul;2018:1632-1635. [doi: 10.1109/EMBC.2018.8512569] [Medline: $\underline{30440706}$ ]

19. Tonino R, Larimer K, Eissen O, Schipperus M. Remote patient monitoring in adults receiving transfusion or infusion for hematological disorders using the VitalPatch and accelerateIQ monitoring system: quantitative feasibility study. JMIR Hum Factors 2019 Dec 02;6(4):e15103 [FREE Full text] [doi: 10.2196/15103] [Medline: 31789596]

20. Areia C, Young L, Vollam S, Ede J, Santos M, Tarassenko L, et al. Wearability testing of ambulatory vital sign monitoring devices: prospective observational cohort study. JMIR Mhealth Uhealth 2020 Dec 16;8(12):e20214 [FREE Full text] [doi: 10.2196/20214] [Medline: $\underline{33325827]}$

21. Breteler MJ, Huizinga E, van Loon K, Leenen LP, Dohmen DA, Kalkman CJ, et al. Reliability of wireless monitoring using a wearable patch sensor in high-risk surgical patients at a step-down unit in the Netherlands: a clinical validation study. BMJ Open 2018 Feb 27;8(2):e020162 [FREE Full text] [doi: 10.1136/bmjopen-2017-020162] [Medline: 29487076]

22. Breteler MJ, KleinJan EJ, Dohmen DA, Leenen LP, van Hillegersberg R, Ruurda JP, et al. Vital signs monitoring with wearable sensors in high-risk surgical patients: a clinical validation study. Anesthesiology 2020 Mar;132(3):424-439 [FREE Full text] [doi: 10.1097/ALN.0000000000003029] [Medline: $\underline{31743149]}$

23. Chan AM, Selvaraj N, Ferdosi N, Narasimhan R. Wireless patch sensor for remote monitoring of heart rate, respiration, activity, and falls. Annu Int Conf IEEE Eng Med Biol Soc 2013;2013:6115-6118. [doi: 10.1109/EMBC.2013.6610948] [Medline: 24111135]

24. Areia C, Vollam S, Piper P, King E, Ede J, Young L, et al. Protocol for a prospective, controlled, cross-sectional, diagnostic accuracy study to evaluate the specificity and sensitivity of ambulatory monitoring systems in the prompt detection of hypoxia and during movement. BMJ Open 2020 Jan 12;10(1):e034404 [FREE Full text] [doi: 10.1136/bmjopen-2019-034404] [Medline: 31932393]

25. Nelson BW, Low CA, Jacobson N, Areán P, Torous J, Allen NB. Guidelines for wrist-worn consumer wearable assessment of heart rate in biobehavioral research. NPJ Digit Med 2020 Jun 26;3:90 [FREE Full text] [doi: 10.1038/s41746-020-0297-4] [Medline: 32613085]

26. Subbe CP, Kinsella S. Continuous monitoring of respiratory rate in emergency admissions: evaluation of the RespiraSense ${ }^{\mathrm{TM}}$ sensor in acute care compared to the industry standard and gold standard. Sensors (Basel) 2018 Aug 17;18(8):2700 [FREE Full text] [doi: 10.3390/s18082700] [Medline: 30126085]

27. Cardiac monitors, heart rate meters, alarms. Association for the Advancement of Medical Instrumentation (AAMI). URL: https://global.ihs.com/doc detail.cfm?document name=AAMI\%20EC13\&item s key=00134307 [accessed 2021-08-09]

28. Kottner J, Audigé L, Brorson S, Donner A, Gajewski BJ, Hróbjartsson A, et al. Guidelines for Reporting Reliability and Agreement Studies (GRRAS) were proposed. J Clin Epidemiol 2011 Jan;64(1):96-106. [doi: 10.1016/j.jclinepi.2010.03.002] [Medline: 21130355]

29. Yasuma F, Hayano J. Respiratory sinus arrhythmia: why does the heartbeat synchronize with respiratory rhythm? Chest 2004 Feb;125(2):683-690. [doi: 10.1378/chest.125.2.683] [Medline: 14769752]

30. Chan AM, Ferdosi N, Narasimhan R. Ambulatory respiratory rate detection using ECG and a triaxial accelerometer. Annu Int Conf IEEE Eng Med Biol Soc 2013;2013:4058-4061. [doi: 10.1109/EMBC.2013.6610436] [Medline: 24110623]

31. Fitzpatrick T. Soleil et Peau. J Med Esthet 1975;2:33-34 [FREE Full text]

32. ixTrend. ixitos. URL: https://www.ixitos.com/en/products/ixtrend/ [accessed 2021-08-09]

33. Davison AC, Hinkley DV. Bootstrap Methods and their Application. Cambridge: Cambridge University Press; Oct 1997.

34. Bland JM, Altman DG. Agreement between methods of measurement with multiple observations per individual. J Biopharm Stat 2007;17(4):571-582. [doi: 10.1080/10543400701329422] [Medline: 17613642]

35. Tiit E. Nonparametric statistical methods. In: Statistics in Medicine. Hoboken, New Jersey: Wiley; May 12, 2000. 
36. R: a language and environment for statistical computing. The R Foundation. URL: https://www.r-project.org/ [accessed 2021-08-09]

\section{Abbreviations}

ANSI/AAMI: American National Standards Institute/Association for the Advancement of Medical Instrumentation

bpm: beats per minute

ECG: electrocardiography

HR: heart rate

LoA: limits of agreement

MAE: mean absolute error

NIHR: National Institute for Health Research

RMSE: root mean square error

rpm: respirations per minute

RR: respiratory rate

$\mathrm{SpO}_{2}$ : peripheral oxygen saturation

STS: sit-to-stand

vHDU: virtual high dependency unit

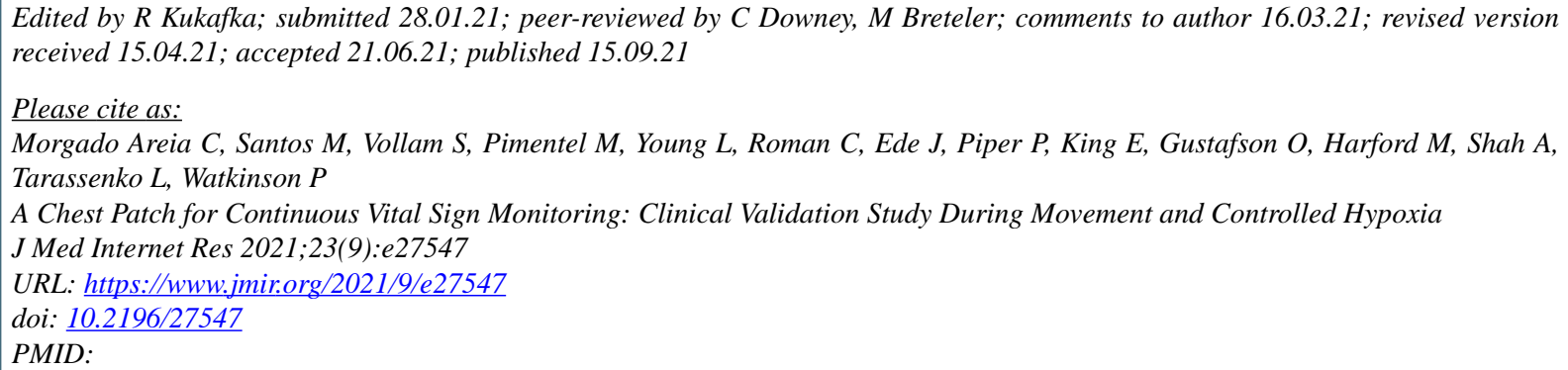

(C) Carlos Morgado Areia, Mauro Santos, Sarah Vollam, Marco Pimentel, Louise Young, Cristian Roman, Jody Ede, Philippa Piper, Elizabeth King, Owen Gustafson, Mirae Harford, Akshay Shah, Lionel Tarassenko, Peter Watkinson. Originally published in the Journal of Medical Internet Research (https://www.jmir.org), 15.09.2021. This is an open-access article distributed under the terms of the Creative Commons Attribution License (https://creativecommons.org/licenses/by/4.0/), which permits unrestricted use, distribution, and reproduction in any medium, provided the original work, first published in the Journal of Medical Internet Research, is properly cited. The complete bibliographic information, a link to the original publication on https://www.jmir.org/, as well as this copyright and license information must be included. 